Landgericht zum christlichen Glauben übergetreten ist. Aus welchen Gründen nunmehr die Hinnahme des bei Ankauf bereits vorhandenen Kunstwerks mit der christlichen Grundeinstellung unvereinbar sein soll, kann nicht nachvollzogen werden. Mangels Anwesenheit in der mündlichen Verhandlung vor dem Senat konnte dies auch dort nicht aufgeklärt werden. Der Vortrag bleibt damit unsubstantiiert und mutet wie eine Schutzbehauptung an.

2. Soweit die Berufung sich gemäß dem Berufungsantrag zu 1 auch gegen die Stattgabe des Unterlassungsantrags, die Plastik zu entsorgen oder zu vernichten, wendet, hat sie auch damit keinen Erfolg.

Die Berufung ist insoweit zwar auch ohne gesonderte Ausführungen im Sinne des $§ 520$ Abs. 3 S. 2 Nr. 2 ZPO zu diesem prozessual eigenständigen Anspruch als zulässig anzusehen. Eine eigenständige Auseinandersetzung erscheint hier ausnahmsweise entbehrlich, da sämtliche von den Klägern geltend gemachten Ansprüche maßgeblich von der Beurteilung der Reichweite des Urheberschutzes an der Plastik sowie etwaiger entgegenstehender wirtschaftlicher Interessen der Beklagten abhängen und die Beklagte sich mit diesen Fragestellungen im Rahmen des Anspruchs auf Reinstallation auseinandergesetzt hat (vgl. BGH, Urt. v. 11.12.2014 - I ZR 8/13 - UsedSoft III).
Die Berufung ist jedoch auch hinsichtlich dieses Antrags unbegründet. Das Landgericht hat zu Recht die für den Unterlassungsanspruch erforderliche Erstbegehungsgefahr insbesondere im Hinblick auf die E-Mail des Geschäftsführers der persönlich haftenden Gesellschafterin der Beklagten vom 16.7.2014 an den damaligen Bevollmächtigten des Klägers zu 2 gestützt. Ebenfalls mit überzeugender Begründung hat es ausgeführt, dass keine das Eigentumsrecht des Klägers zu 2 sowie das Urheberrecht des Klägers zu 1 überwiegenden Interessen der Beklagten dargelegt wurden, die Plastik zu vernichten oder zu entsorgen. Zur Vermeidung von Wiederholungen wird insoweit auf die überzeugenden Ausführungen des Landgerichts Bezug genommen.

3. Soweit mit der Berufung zudem die abgewiesene Widerklage weiterverfolgt wird, hat die Berufung auch insoweit keinen Erfolg. Die Unbegründetheit der Widerklage folgt reziprok aus den Ausführungen unter Ziff. 1 zur Begründetheit des Anspruchs auf Reinstallation.

\title{
Otto Mueller - Polizeiliche Beschlagnahme von Fälschungen
}

VG Berlin Urteil vom 01.12.2016 - 1 K 236.13

Fälschungen sind Nachbildungen, die in Täuschungsabsicht hergestellt wurden und können damit als taugliche Tatmittel einer Betrugsstraftat beschlagnahmt werden und bleiben. (Leitsatz der Redaktion)

\section{Tatbestand}

[1] Die Beteiligten streiten über die Sicherstellung von 14 Farbkreidezeichnungen.

[2] Am 24. Januar 2013 wurden vom Beklagten im Rahmen einer Fahrzeugüberprüfung bei Herrn M... 14, jeweils mit "Otto Müller" signierte Farbkreidezeichnungen sichergestellt. Die Sicherstellung erfolgte zunächst auf der Grundlage der Strafprozessordnung. Mit Schreiben seines Bevollmächtigten vom 31. Januar 2013 teilte der Kläger mit, er sei Eigentümer der Zeichnungen. Nach Freigabe der Bilder durch die Staatsanwaltschaft Berlin am 6. Februar 2013 wurde der Kläger mit Schreiben des Beklagten vom 3. Juni 2013 über eine nach § 38 Nr. 1 des Allgemeinen Sicherheits- und Ordnungsgesetzes erfolgte Sicherstellung unterrichtet. Gegen die Sicherstellung legte der Kläger am 3. Juli 2013 Widerspruch ein, der mit Widerspruchsbescheid vom 9. Juli 2013 als unbegründet zurückgewiesen wurde.

[3] Der Kläger trägt vor, er habe die Zeichnungen auf einem Flohmarkt erworben. Ob es sich um authentische Werke des Künstlers Otto Müller handle, könne er mangels entsprechendem Sachverstand nicht beurteilen. Er habe die Bilder gekauft, weil sie inm gefallen haben. Eventuell werde er nicht alle Arbeiten behalten, beabsichtige aber jedenfalls nicht, gefälschte Kunstwerke als echt zu veräußern. Er sei bereit, eine Kennzeichnung anzubringen, wonach die Echtheit nicht belegt sei.

[4] Der Kläger beantragt,

[5] den Bescheid vom 3. Juni 2013 in Gestalt des Widerspruchsbescheids vom 9. Juli 2013 aufzuheben und die 14 sichergestellten Bilder herauszugeben. 
[6] Der Beklagte beantragt,

[7] die Klage abzuweisen.

[8] Er verteidigt den ergangenen Bescheid.

[9] Die Kammer hat durch die Berichterstatterin Beweis erhoben durch Vernehmung des vom Beklagten als Sachverständigen herangezogenen D... als sachverständigen Zeugen.

[10] Wegen der weiteren Einzelheiten des Sach- und Streitstandes wird auf den Inhalt der Gerichtsakte sowie die vom Beklagten vorgelegten Verwaltungsvorgänge Bezug genommen.

\section{Entscheidungsgründe}

[11] Die Kammer konnte gem. § 101 Abs. 2 der Verwaltungsgerichtsordnung - VwGO - ohne mündliche Verhandlung entscheiden, weil die Beteiligten sich damit einverstanden erklärt haben.

[12] Die Klage hat keinen Erfolg. Der Kläger kann weder die Aufhebung des Bescheids vom 3. Juni 2013 in Gestalt des Widerspruchsbescheids vom 9. Juli 2013 (I.) noch die Herausgabe der sichergestellten Farbkreidezeichnungen (II.) verlangen.

[13] I. Soweit der Kläger die Aufhebung der Sicherstellungsanordnung begehrt, ist die Klage als Anfechtungsklage gem. § 42 Abs. 1 Alt. 1 VwGO statthaft und auch im Übrigen zulässig. Bei dem Schreiben vom 3. Juni 2013 handelt es sich insbesondere trotz der Formulierung als Informationsschreiben um einen Verwaltungsakt iSd § 35 S. 1 Verwaltungsverfahrensgesetz - VwVfG - iVm § 1 Abs. 1 Berliner Verwaltungsverfahrensgesetz - BlnVwVfG. Unter Berücksichtigung der gesamten Umstände - namentlich der zuvor mit Schreiben vom 11. Februar 2013 erfolgten Anhörung und der Äußerungen der Klägerseite vom 5. März 2013 und 24. April 2013 - muss der Schluss gezogen werden, dass die mit einer Rechtsbehelfsbelehrung versehene Mitteilung des Beklagten vom 3. Juni 2013 auf das Setzen einer Rechtsfolge, nämlich die Begründung eines Verwahrungsverhältnisses iSd § 39 ASOG gerichtet war.

[14] Die Sicherstellung ist rechtmäßig erfolgt und der Kläger insoweit nicht in seinen Rechten verletzt (§ 113 Abs. 1 S. 1 VwGO).

[15] Rechtsgrundlage der Maßnahme ist § 38 Nr. 1 ASOG. Danach können die Ordnungsbehörden und die Polizei eine Sache sicherstellen, um eine gegenwärtige Gefahr abzuwehren. Unter einer Gefahr ist eine Sachlage zu verstehen, die bei ungehindertem Geschehensablauf des zu erwartenden Geschehens in überschaubarer Zukunft mit hin- reichender Wahrscheinlichkeit zu einem Schaden für die Schutzgüter der öffentlichen Sicherheit und Ordnung führen wird (vgl. Urteile der Kammer vom 16. September 2011 - 1 K 312.10, juris, Rn. 15 und vom 6. Mai 2010 - 1 K 927.09, juris, Rn. 14 mwN). Die öffentliche Sicherheit umfasst die Rechtsordnung als Ganzes, die Individualrechtsgüter und den Staat und seine Einrichtungen (BVerfG, Beschl. v. 14. Mai 1985 - 1 BvR 233/81 u.a. juris, Rn. 77; BVerwG, Urt. v. 21. April 1989 - 7 C 50.88, juris, Rn. 15). Die Gefahrenlage braucht nicht in einer Eigenschaft der sicherzustellenden Sache begründet sein (wie beispielsweise bei Waffen), sondern kann sich aus dem Verhalten ihres Besitzers ergeben (Urt. der Kammer v. 16. September 2011 - 1 K 312.10, juris, Rn. 15). Gegenwärtig ist eine Gefahr, wenn der Eintritt des schädigenden Ereignisses bereits begonnen hat oder mit an Sicherheit grenzender Wahrscheinlichkeit unmittelbar bevorsteht (Urt. der Kammer v. 6. Mai 2010 1 K 927.09, juris, Rn. 18; Pewestorf in: Pewestorf/Söllner/ Tölle, Praxishandbuch Polizei- und Ordnungsrecht, 2013, Kap. 1, Rn. 28). Auch bei Anwendung des qualifizierten Gefahrbegriffs (Merkmal der Gegenwärtigkeit als zusätzliche Eingriffsvoraussetzung) ist hinsichtlich der Wahrscheinlichkeit der Gefahrenverwirklichung eine differenzierte Betrachtung geboten. Je schwerer der Schaden ist, der einzutreten droht, desto geringer sind die Anforderungen, die an die Wahrscheinlichkeit des Schadenseintritts zu stellen sind (OVG Lüneburg, Urt. v. 25. Juni 2015 - 11 LB 34/14, juris, Rn. 34; VG Braunschweig, Beschl.v. 19. Oktober 2006 - 5 B 284/06, juris, Rn. 23 mwN).

[16] Nach diesen Maßgaben war vorliegend zum Zeitpunkt der Sicherstellung eine gegenwärtige Gefahr für die öffentliche Sicherheit gegeben. Es musste befürchtet werden, dass die sichergestellten Farbkreidezeichnungen, bei denen es sich um Kunstfälschungen handelt (1.), künftig zur Begehung von Betrugsstraftaten genutzt werden (2.). Die Sicherstellung ist ermessensfehlerfrei erfolgt und nicht unverhältnismäßig (3.).

[17] 1. Die 14 Zeichnungen sind keine authentischen Werke des Künstlers Otto Müller (1874 -1930). Die Kammer legt insoweit das Ergebnis der Begutachtung durch die vom Beklagten beauftragten Kunstsachverständigen D... und F... zugrunde, an deren Richtigkeit zu zweifeln kein Anlass besteht. Herr D... ist Kunsthistoriker und Kustos der Abteilung Malerei, Skulptur und Medienkunst des 19. und 20. Jahrhunderts am Museum Folkwang in Essen. Zu dem Künstler Otto Müller hat er mehrfach publiziert und u.a. ein Werkverzeichnis der Gemälde und auf Papier ausgeführten Arbeiten Otto Müllers veröffentlicht. Herr K... war als Verfasser eines Werkverzeichnisses der Graphik Otto Müllers und Kunsthändler ebenfalls sachkundig. Zweifel am Sachverstand des Herrn K... infolge des Freispruchs des Herrn Edgar M..., dessen Arbeiten Herr K... als Fälschungen qualifiziert hatte, bestehen nicht. Der Freispruch war erfolgt, nachdem die Echtheit der betreffenden Werke von 
anderen Experten nicht ausgeschlossen werden konnte; zu einem späteren Zeitpunkt hat Herr M... jedoch eingeräumt, Kunstwerke gefälscht zu haben und wurde von der Kriminalpolizei überführt.

[18] Herr D... wurde gem. § 87 Abs. 3 VwGO durch die Berichterstatterin als sachverständiger Zeuge über seine am 1. März 2013 im Beisein des Klägervertreters erfolgte Begutachtung befragt. Der Zeuge hat ausgesagt, er identifiziere die sichergestellten Zeichnungen eindeutig als (primitive) Fälschungen. Dies ergebe sich zum einen aus den verwendeten Materialien; sowohl die hier eingesetzten Pastellkreiden als auch die Papierart und die Formate der Blätter seien völlig untypisch für den Künstler Otto Müller. Gleiches gelte für die Signatur, die von Otto Müller in der Regel mit Bleistift ausgeführt wurde, bei den streitgegenständlichen Zeichnungen jedoch mit Kreide oder Farbstift. Schließlich erscheine der Malstil zaghaft und lasse eine große Unsicherheit mit dem Sujet erkennen. Den echten Werken Otto Müllers läge eine deutlich geschlossenere Komposition zugrunde. In eine Szene hineinragende, abgeschnittene Büsche wie z.B. bei Bl. 12 der Ablichtungen seien absolut untypisch für Otto Müller. Schließlich dürften die hier streitgegenständlichen Zeichnungen - ihrem Sinn und Zweck nach Vorzeichnungen - unter Zugrundelegung der Arbeitsweise Otto Müllers gar nicht existieren, weil der Künstler für die Erstellung seiner Lithographien und Gemälde prinzipiell keine Vorzeichnungen angefertigt hat. Die auf BI. 1, 2, 3 und 5 der Abbildungen wiedergegebenen Zeichnungen sind ferner im Werksverzeichnis der Gemälde und auf Papier ausgeführten Arbeiten, das D... erstellt hat, als Fälschungen publiziert.

[19] Nach den Feststellungen des sachverständigen Zeugen ist weiterhin auszuschließen, dass die Arbeiten als bloße Nachbildungen ohne Täuschungsabsicht angesehen werden können. Aus der aufwendig ausgeführten, auffällig in Szene gesetzten Signatur müsse der Schluss gezogen werden, dass die Arbeiten vorgeben sollen, echte Werke des Künstlers Otto Müller zu sein.

[20] Schließlich handelt es sich - ungeachtet der Frage, welche rechtliche Relevanz dem zukommen würde - nach der Aussage des sachverständigen Zeugen auch nicht um wertvolle Fälschungen aus der Hand des berühmten Fälschers Edgar M.... Dem angekündigten Beweisantrag der Klägerseite, die Tochter des Herrn M... als Zeugin zu laden, musste vorliegend nicht entsprochen werden. Der Beweisantrag ist bereits nicht hinreichend bestimmt, weil nicht eindeutig erkennbar ist, welche Tatsache durch die Einvernahme der Frau M... bewiesen werden soll. Soweit es entsprechend dem schriftsätzlichen Vortrag um die Behauptung gehen soll, Edgar M... sei letzter bekannter Eigentümer der (vom Kläger als echt angesehenen) Zeichnungen gewesen, kann dies vorliegend als wahr unterstellt werden (vgl. § 244 Abs. 3 S. 2 Alt. 7 der Straf- prozessordnung); für die Frage der Echtheit der Arbeiten und die Eignung zur Begehung von Betrugsstraftaten ist es ohne Belang, ob Herr M... früher einmal Eigentümer der Bilder war. Eine Vorabentscheidung über den Beweisantrag durch Beschluss gem. § 86 Abs. 2 VwGO hatte vorliegend nicht zu erfolgen, weil diese Pflicht nur für in der mündlichen Verhandlung gestellte, unbedingte Beweisanträge gilt. Verzichtet ein Beteiligter - wie hier der Kläger mit Schriftsatz vom 13. September 2016 - nach schriftsätzlicher Ankündigung eines Beweisantrages auf die Durchführung einer mündlichen Verhandlung ( $\$ 101$ Abs. 2 VwGO), so hat er sich der Möglichkeit zur Geltendmachung des Anspruchs auf Vorabentscheidung aus $\S 86$ Abs. 2 VwGO begeben (BVerwG, Beschl. v. 6. September 2011 - 9 B 48/11, juris, Rn. 10, mwN; OVG Münster Beschl. v. 24. April 2012 - 1 A 2243/10, BeckRS 2012, 49994).

[21] Ausweislich des Vernehmungsprotokolls des Beklagten vom 25. Januar 2013 kam der weitere Sachverständige Herr K... nach Inaugenscheinnahme der Zeichnungen ebenfalls zu dem Ergebnis, dass die Arbeiten mit „absoluter Sicherheit" nicht von dem Künstler Otto Müller stammen, sondern es sich um dilettantische Fälschungen handelt. Da Motive und Signaturen der Werke Otto Müllers nachgeahmt werden, wurden die Zeichnungen von Herrn K... als Fälschungen mit Täuschungsabsicht qualifiziert. Die auf BI. 1 und 7 der Ablichtungen sowie auf BI. 1, 2, 3 und 5 wiedergegebenen Zeichnungen wurden von Herrn K... zudem bereits bei früheren Begutachtungen in den Jahren 2012 sowie 1970 bis 1975 als Fälschungen identifiziert und teilweise (BI. 1, 2, 3 und 5 der Ablichtungen) in sein Fälschungsarchiv aufgenommen.

[22] 2. Vorliegend besteht ein erhebliches Risiko, dass die Fälschungen in betrügerischer Absicht als authentische Werke auf dem Kunstmarkt veräußert werden könnten und dadurch das Vermögen eines Erwerbers erheblich geschädigt werden würde.

[23] a) Die streitgegenständlichen Zeichnungen sind taugliche Tatmittel einer Betrugsstraftat. Wie soeben ausgeführt, handelt es sich nach den Feststellungen der Sachverständigen nicht um bloße Nachbildungen ohne Fälschungscharakter. Durch die aufwendig ausgeführte Signatur soll vielmehr der Eindruck einer Urheberschaft des Künstlers Otto Müller erweckt werden. Die streitgegenständlichen Arbeiten sind auch nicht zur Täuschung schlechthin ungeeignet, weil die fehlende Echtheit offenkundig wäre. Den Ausführungen des sachverständigen Zeugen D... zufolge könnte zwar ein Galerist mit diesen Fälschungen wahrscheinlich nicht getäuscht werden; einem privaten Käufer könnte jedoch gegebenenfalls erfolgreich vorgespiegelt werden, dass es sich um authentische Werke Otto Müllers handelt. Dafür spricht auch, dass vier der insgesamt 14 streitgegenständlichen Zeichnungen, die auf dunklen Karton aufgezogenen Arbeiten (BI. 1, 2, 3 und 5 der Ab- 
lichtungen), dem Sachverständigen Karsch in den 1970er Jahren bereits von drei unterschiedlichen Personen zur Begutachtung und Feststellung der Echtheit vorgelegt wurden. Obwohl die Zeichnungen von H... jeweils als Fälschungen identifiziert und in sein Fälschungsarchiv aufgenommen wurden, gelangten diese wieder auf den Markt und wurden als möglicherweise echte Werke Otto Müllers erworben.

[24] b) Für die Befürchtung, die Zeichnungen könnten in nächster Zukunft als authentische Werke Otto Müllers zum Verkauf angeboten werden, spricht vorliegend, dass bei den Arbeiten Notizzettel sichergestellt wurden, denen sich Angaben zu möglichen Werten der Bilder und einem Verkaufsprozedere entnehmen lassen. So finden sich dort die Notizen: „Kreidezeichnungen ca. 45.000, dann nach Gebot, 38 × 30 - 38 × 28, bei Anwalt Gebot abgeben!“ sowie "Rupfen $800.000-1,2$ M“. Der Vortrag des Klägers, ein - nicht namentlich bezeichneter - Bekannter habe mögliche Werte lediglich aus eigener Neugier recherchiert und seine Ergebnisse dokumentiert, dies habe jedoch nicht der Vorbereitung eines Verkaufs gedient, ist unglaubhaft. Insbesondere konnte nicht erklärt werden, wie der Vermerk „ca. 45.000, dann nach Gebot" bzw. „bei Anwalt Gebot abgeben!" anders als ein mögliches Vorgehen zur Veräußerung der Bilder zu verstehen sein soll. Für das Bestehen von Verkaufsabsichten spricht auch, dass die Zeichnungen dem Vortrag der Klägerseite zufolge bereits einem Kunstvermittler präsentiert wurden. Auf ein bloßes Besitzen der Bilder aus einem reinen Affektionsinteresse heraus hat sich der Kläger damit gerade nicht beschränkt. Zwar ist es denkbar, dass der konkrete Präsentationstermin, nach dem die Bilder sichergestellt wurden, lediglich der Inaugenscheinnahme und Bewertung dienen sollte und ein konkreter Verkauf (noch) nicht geplant war, wie der Kläger vorträgt. Dadurch wird jedoch die Befürchtung, die Zeichnungen könnten demnächst unter Verwirklichung des Betrugstatbestandes veräußert werden, nicht ausgeräumt. Dass eine Veräußerung der Zeichnungen grundsätzlich geplant ist, ergibt sich auch aus dem Schreiben des Klägervertreters vom 31. Januar 2013, in dem dieser mitteilt, der zukünftige Erwerber/Vermittler wolle die Bilder begutachten lassen.

[25] c) Die Gefahr ist auch gegenwärtig. Angesichts des möglichen erheblichen Vermögensschadens - echte Zeichnungen Otto Müllers haben dem Sachverständigen D... zufolge einen Wert von $65.000 €$ bis $200.000 €$ und darüber - sind die Anforderungen an die zeitliche Nähe des Schadenseintritts vorliegend nicht zu überspannen. Wie sich aus der Einlassung des Sachverständigen K... ergibt, wurden die Arbeiten auf dunklem Karton (BI. 1, 2, 3 und 5 der Ablichtungen) im Zeitraum von nur fünf Jahren von 1970 bis 1975 mindestens drei Mal (Bl. 5) bzw. zwei Mal (BI. 1, 2 und 3) veräußert. Im Jahr 2012 wurden Herrn K... die auf BI. 1 und 7 der Ablichtungen wiedergegebenen Zeich- nungen (erneut) zur Begutachtung durch einen Galeristen vorgelegt. Somit wäre auch vorliegend jederzeit mit einer Veräußerung der Bilder zu rechnen.

[26] 3. Der Beklagte hat die ihm durch $\S 38$ ASOG eingeräumte Befugnis ermessensfehlerfrei ausgeübt; Ermessensfehler sind nicht vorhanden (§ 114 VwGO). Die Verhältnismäßigkeit der Maßnahme ist gewahrt (§ 11 ASOG). Ein milderes Mittel zur Vermeidung von Betrugsstraftaten durch Täuschung potentieller Erwerber über die Echtheit der Zeichnungen ist nicht ersichtlich. Die bloße Veröffentlichung der Arbeiten als Fälschungen ist zur Zweckerreichung nicht ebenso geeignet. Die Zeichnungen BI. 1, 2, 3 und 5 der Abbildungen sind seit 2003 im Werksverzeichnis des D... als Fälschungen veröffentlicht. Dadurch konnte in der Vergangenheit jedoch nicht verhindert werden, dass die Zeichnungen auf dem Kunstmarkt angeboten werden, wie insbesondere die Vorlage der Zeichnung BI. 1 zur Begutachtung durch Herrn K... im Jahr 2012 belegt. Auch durch eine Kennzeichnung auf der Rückseite kann nicht mit hinreichender Sicherheit verhindert werden, dass die Zeichnungen - unter Unterdrückung der Kennzeichnung - künftig als echte Kunstwerke angeboten werden. Der Beklagte hat hierzu ausgeführt, in der Vergangenheit seien in mindestens zwei Fällen durch das LKA angebrachte Kennzeichnungen wieder entfernt worden. Dabei sei für das bloße Auge nicht sichtbar - ein Stempel mit einer Rasierklinge abgekratzt und eine schriftliche Markierung mit chemischen Mitteln entfernt worden. Die Tatsache, dass derartige Maßnahmen relativ aufwendig sind, räumt angesichts des erheblichen Wertes echter Werke Otto Müllers dieses Risiko nicht aus. Denkbar ist es außerdem auch, die Blätter auf Pappe aufzuziehen und mit dieser fest zu verkleben. Für einen möglichen Erwerber könnte es so den Anschein haben, als handle es sich um eine kartonartige Rückwand, die vom Künstler selbst oder einem Sammler angebracht wurde. Auch durch ein Abtrennen der Signatur kann nicht ausgeschlossen werden, dass das Werk als „im Stile Otto Müllers" und "möglicherweise echt" veräußert wird, wie der Sachverständige D... ausgeführt hat.

[27] Die von der Klägerseite vorgeschlagene Abgabe einer Zusicherung, die Werke nicht bzw. nur mit Zustimmung des Beklagten zu veräußern oder als Pfand einzusetzen einschließlich einer entsprechenden Verfügung in seinem Testament ist zur Zweckerreichung ebenfalls nicht gleich geeignet. Diese Maßnahme verspricht nicht das gleiche $\mathrm{Maß}$ an Sicherheit und Schutz des Rechtsverkehrs vor Betrugsstraftaten wie es eine Sicherstellung der Fälschungen gewährleistet.

[28] Im Rahmen der Interessenabwägung ist schließlich zu berücksichtigen, dass der Kläger selbst mitgeteilt hat, die Zeichnungen sehr günstig erworben zu haben, wobei die Höhe des Kaufpreises von ihm weder genannt noch 
nachgewiesen wurde. Aus der Stellungnahme des sachverständigen Zeugen D... ergibt sich, dass die Zeichnungen weder einen materiellen noch einen künstlerischen Wert aufweisen. Das damit im Wesentlichen verbleibende immaterielle Interesse des Klägers am Besitz und an der Sammlung der Zeichnungen wurde vom Beklagten zu Recht als nachrangig gegenüber dem Interesse am Schutz des Rechtsverkehrs vor Betrugsstraftaten eingestuft.

[29] II. Da die Sicherstellung rechtmäßig ist, hat der Kläger keinen Herausgabeanspruch im Wege der Vollzugsfolgenbeseitigung gem. § 113 Abs. 1 S. 2 VwGO.
[30] Der Kläger kann die Herausgabe schließlich auch nicht gem. § 41 Abs. 1 S. 1 ASOG verlangen, weil die Voraussetzungen für die Sicherstellung nicht weggefallen sind. Eine gegenwärtige Gefahr der Begehung von Betrugsstraftaten mithilfe der Fälschungen besteht weiterhin und würde sich im Fall einer Herausgabe der Bilder an den Kläger erneut aktualisieren ( $\$ 41$ Abs. 1 S. 3 ASOG).

[31] Ob die Zeichnungen an den Kläger, der seine Eigentümerstellung nicht belegt hat, zurückzugeben wären oder an Herrn M..., bei dem die Bilder sichergestellt wurden (vgl. § 41 Abs. 1 S. 2 ASOG), kann damit offen bleiben.

\section{Temporäre Kunst vor der Frauenkirche Dresden}

Verwaltungsgericht Dresden, Beschluss vom 15.02.2017, 12 L 190/17

Eine von der Stadt erteilte Sondernutzungserlaubnis zur Aufstellung eines Kunstwerkes kann einen Einzelnen nicht in seinen Rechten verletzten. Ein Bürger ist nicht davor gesetzlich geschützt, dass der Anblick von Kunst bei ihm „anstößige Wertung erregt“ (Leitsatz der Redaktion)

\section{Gründe}

- Der Antragsteller begehrt im einstweiligen Anordnungsverfahren die Verpflichtung der Antragsgegnerin, das auf dem NMarkt in D vor der F-Kirche aufgestellte Kunstwerk "Monument" zu entfernen.

Bei dem temporären Kunstwerk "Monument" des Künstlers $\mathrm{H}$ handelt es sich um die Installation von drei Bussen, die auf einem Sockel auf dem N-Markt hochkant aufgestellt sind. Diese sollen an drei Linienbusse erinnern, die in Aleppo zum Schutz vor Scharfschützen aufgestellt worden waren. Die Installation ist Teil des Kulturfestivals "Am Fluss. Zu Kulturen des Ankommens" im Rahmen der Initiative W. Die von den Museen der Stadt D/Kunsthaus D beantragte Sondernutzungserlaubnis für die Nutzung einer Teilfläche des N-Marktes für die Aufstellung des Kunstwerkes hat die Antragsgegnerin am 2. Februar 2017 erteilt.

Der Antragsteller hat am 8. Februar 2017 den vorliegenden Antrag auf Gewährung einstweiligen Rechtsschutzes gestellt. Er ist der Auffassung, dass es - insbesondere auch im Gedenken an die Opfer des 13. Februar 1945 - unangemessen und respektlos sei, diese „Kunstart" vor der D F-Kirche aufzustellen. In der allgemeinen Stimmungslage sei sie eine Provokation. Das Gedenken an die Opfer des 2. Weltkriegs werde mit der aktuellen Situation in Aleppo vermischt. Die "Kunst" werde Tag und Nacht von Polizisten bewacht. Dafür würden Steuergelder aufgewendet.

Die Antragsgegnerin ist dem Antrag entgegen getreten und verweist darauf, dass nicht erkennbar sei, inwiefern der Antragsteller in eigenen Rechten verletzt sei. Ob eine Sondernutzung erteilt werden könne, bemesse sich ausschließlich nach straBenrechtlichen Kriterien, d.h. nach solchen Belangen, welche einen Bezug zur öffentlichen Straße hätten. Dies seien Belange zum Schutz der öffentlichen Straßenlage unter Aufrechterhaltung der Sicherheit und Leichtigkeit des Verkehrs sowie Belange der öffentlichen Sicherheit und Ordnung. Straßenfremde Kriterien könnten nicht zur Beurteilung über die Zulässigkeit des Sondernutzungsantrages herangezogen werden. Insbesondere obliege es nicht dem Straßenbaulastträger, über den Inhalt einer Kunstausübung zu befinden.

Der Antrag hat keinen Erfolg. Er ist bereits unzulässig.

Nach § 123 Abs. 1 VwGO kann das Gericht, auch schon vor Klageerhebung, eine einstweilige Anordnung in Bezug auf den Streitgegenstand treffen, wenn die Gefahr besteht, dass durch eine Veränderung des bestehenden Zustands die Verwirklichung eines Rechts des Antragstellers vereitelt oder erschwert werden könnte. Einstweilige Anordnungen sind auch zur Regelung eines vorläufigen Zustands in Bezug auf ein streitiges Rechtsverhältnis zulässig, wenn diese Regelung, um wesentliche Nachteile abzuwenden oder drohende Gewalt zu verhindern oder aus anderen Gründen nötig erscheint. Der geltend gemachte Anspruch (Anordnungsanspruch) und die Notwendigkeit der vorläufigen Regelung (Anordnungsgrund) sind glaubhaft zu machen ( $§ 123$ Abs. 3 VwGO iVm § 920 Abs. 2 ZPO).

Der Antrag ist unzulässig. Dem Antragsteller fehlt es an der erforderlichen Antragsbefugnis (§ 42 Abs. 2 VwGO analog). 